BULLETIN OF THE

AMERICAN MATHEMATICAL SOCIETY

Volume 77, Number 1, January 1971

\title{
ISOMORPHISM THEORY OF CONGRUENCE GROUPS
}

\author{
BY ROBERT E. SOLAZZI
}

Communicated by I. N. Herstein, May 18, 1970

This note is an announcement of four theorems I proved in [5][9] on the isomorphisms of the linear, symplectic, and unitary congruence groups. A sketch of the proofs is given.

Notation. Let $V$ be an $n$-dimensional vector space over the field $F$, $n \geqq 2$. For $\sigma \in G L_{n}(V)$, let $\check{\sigma}$ denote the contragredient of $\sigma$ (inverse of the transpose). Let-denote the natural map of $G L_{n}(V)$ onto $P G L_{n}(V)$; for any subset $S$ of $G L_{n}(V), \bar{S}$ is the image of $S$ in $P G L_{n}(V)$ under the-map.

A transvection $\tau$ is a linear transformation of determinant one which fixes all vectors of some hyperplane, called the proper hyperplane of $\tau$. If $\tau \neq 1$ then $(\tau-1) V$ is a line called the proper line of $\tau$. An element $\bar{\tau}$ of $P G L_{n}(V)$ is called a (projective) transvection if $\tau$ is a transvection. The proper line and proper hyperplane of $\bar{\tau}$ are defined as the proper line and hyperplane of $\tau$.

Congruence groups. Again let $V$ be an $n$-dimensional vector space over $F, n \geqq 2$; let $o$ be any integral domain with quotient field $F$. An $o$-module $M \subset V$ is bounded if there is an o-linear isomorphism of $M$ into some free $o$-module of finite dimension. Assume $M$ is a bounded o-module with $F M=V$. We define the integral linear group $G L_{n}(M)$ as $\left\{\sigma \in G L_{n}(V) \mid \sigma M=M\right\}$. The integral symplectic group $S p_{n}(M)$ is $\left\{\sigma \in \operatorname{Sp}_{n}(V) \mid \sigma M=M\right\}$ and is only defined for even $n$.

Let $f(x, y)$ be a nondegenerate hermitian form on $V$ whose involution maps the domain on to itself; the integral unitary group $U_{n}(M, f)$ is defined as $U_{n}(M, f)=\left\{\sigma \in U_{n}(V, f) \mid \sigma M=M\right\}$.

Now let $a$ be a nonzero ideal in 0 . Consider the groups

$$
\begin{aligned}
& G L_{n}(M ; a)=\left\{\sigma \in G L_{n}(M) \mid(\sigma-1) M \subset a M\right\}, \\
& S L_{n}(M ; a)=G L_{n}(M ; a) \cap S L_{n}(V)
\end{aligned}
$$

and let $T L_{n}(M ; a)$ be the group generated by all transvections in $G L_{n}(M ; a)$.

Now put $S p_{n}(M ; a)=\left\{\sigma \in S p_{n}(M) \mid(\sigma-1) M \subset a M\right\}$ and let

AMS 1970 subject classifications. Primary 20H05, 15A63; Secondary 15A57.

Key words and phrases. Linear group, symplectic, unitary group, congruence group, transvection, semisimilitute, isotropic line, collineation.

Copyright (c) 1971, American Mathematical Society 
$T S p_{n}(M ; a)$ be the group generated by all transvections in $S P_{n}(M ; a)$.

We say a group $\Delta$ is a linear congruence group if $G L_{n}(M) \supset \Delta$ $\supset T L_{n}(M ; a)$. We will call $\Delta$ a symplectic congruence group if $S p_{n}(M) \supset \Delta \supset T S p_{n}(M ; a)$.

Let

$$
\begin{aligned}
& U_{n}(M, f, a)=\left\{\sigma \in U_{n}(M, f) \mid(\sigma-1) M \subset a M\right\}, \\
& U_{n}^{+}(M, f, a)=U_{n}(M, f, a) \cap S L_{n}(V)
\end{aligned}
$$

and let $T_{n}(M, f, a)$ be the group generated by all transvections in $U_{n}(M, f, a)$. We say a group $\Delta$ is a unitary congruence group if $U_{n}(M, f) \supset \Delta \supset T_{n}(M, f, a)$.

Projective congruence groups. Now let $\Delta$ be any one of the above linear, symplectic, or unitary congruence groups. The projective congruence group corresponding to $\Delta$ is the group $\bar{\Delta}$, the image of $\Delta$ under the- map of $G L_{n}(V)$ onto $P G L_{n}(V)$.

Main theorems. Let $\Delta$ be any one of the linear, symplectic, or unitary congruence groups. If $\Delta$ is a linear congruence group then for every line $L$ and hyperplane $H$ of $V$ with $L C H$ there is a nontrivial transvection in $\Delta$ with proper line $L$ and proper hyperplane $H$. If $\Delta$ is a symplectic or a unitary congruence group then for every isotropic line $L$ of $V$ there is a nontrivial transvection in $\Delta$ with proper line $L$. Similar statements then obviously hold for projective transvections in the projective congruence group $\bar{\Delta}$.

Now consider an automorphism $\Lambda$ of $\bar{\Delta}$. Assume $n \geqq 3$ in the linear case, $n \geqq 6$ in the symplectic case, and that char $F \neq 2$ and $f(x, y)$ has Witt index at least 3 in the unitary case. Using group-theoretical properties of the double centralizer of a projective-transvection, in all cases (linear, symplectic or unitary) we are able to grouptheoretically distinguish the projective transvections in $\bar{\Delta}$ from the other transformations of $\bar{\Delta}$. From this it follows that $\Lambda$ maps any projective transvection to a projective transvection again.

If $\Delta$ is a linear congruence group we then obtain a bijection of the set of lines and hyperplanes of $V$ such that either the set of lines is mapped onto the set of lines or the set of lines is mapped onto the set of hyperplanes. In either case we have a bijection which satisfies the hypothesis of the fundamental theorem of projective geometry and therefore the bijection is induced identically from either a semilinear isomorphism $g$ of $V$ onto $V$ or a semilinear isomorphism $h$ of $V$ onto its dual $V^{\prime}$. It then follows that either $\Lambda \bar{\sigma}=\left(g \sigma g^{-1}\right)-$ for all $\bar{\sigma} \in \bar{\Delta}$, or that $\Lambda \bar{\sigma}=\left(h^{-1} \check{\sigma} h\right)-$ for all $\bar{\sigma} \in \bar{\Delta}$. 
If $\Delta$ is a symplectic congruence group the automorphism $\Lambda$ induces a bijection of the set of lines of $V$ onto itself. We may again apply the fundamental theorem, conclude the bijection is induced from a semilinear isomorphism $g$ of $V$ onto $V$ and then show $\Lambda \bar{\sigma}=\left(g \sigma g^{-1}\right)^{-}$ $\forall \bar{\sigma} \in \bar{\Delta}$. In fact $g$ is a symplectic semisimilitude; that is $B(g x, g y)$ $=\lambda B(x, y)^{u}$ for all $x, y$ in $V$ where $B(x, y)$ is the alternating bilinear form defined on $V, \lambda$ is a scalar in $F$ independent of $x$ and $y$, and $u$ is the field automorphism associated with the semilinear map $g$.

Finally if $\Delta$ is a unitary congruence group, the automorphism $\Lambda$ of $\bar{\Delta}$ again maps projective transvections to projective transvections and so we obtain a bijection of the set of isotropic lines of $V$ onto itself. Extending this bijection to all the lines of $V$ and again applying the fundamental theorem of projective geometry, we show $\Lambda \bar{\sigma}=\left(g \sigma g^{-1}\right)-$ $\forall \bar{\sigma} \in \bar{\Delta}$ where $g$ is some semilinear isomorphism of $V$ onto $V$. As in the symplectic case one easily sees $g$ is a unitary semisimilitude. That is, $f(g x, g y)=\lambda f(x, y)^{u}$ for all $x, y$ in $V$ with $\lambda$ a scalar in $F, u$ the field automorphism of $g$, and $f(x, y)$ the hermitian form.

Having determined all the automorphisms of the projective congruence groups, we obtain as corollaries (under the same assumptions as above ) the following three theorems.

THEOREM 1. Let $\Delta$ be a linear congruence group and $\Lambda$ an automorphism of $\Delta$. Then either

(i) there is a semilinear isomorphism $g$ of $V$ onto $V$ and a homomorphism $\chi$ of $\Delta$ into $F$ such that

$$
\Lambda \sigma=\chi(\sigma) \cdot g \sigma g^{-1} \quad \text { for all } \sigma \in \Delta,
$$

or (ii) there is a semilinear isomorphism $h$ of $V$ onto the dual space $V^{\prime}$ and a homomorphism $\chi$ of $\Delta$ into the field $F$ such that

$$
\Lambda \sigma=\chi(\sigma) \cdot h^{-1} \check{\sigma} h \quad \text { for all } \sigma \in \Delta .
$$

THEOREM 2. Let $\Delta$ be a symplectic congruence group and $\Lambda$ an automorphism of $\Delta$. Then there is a semilinear isomorphism $g$ of $V$ onto $V$ (in fact $g$ is a semisimilitude) and a homomorphism $\chi$ of $\Delta$ into \pm 1 such that

$$
\Lambda \sigma=\chi(\sigma) \cdot g \sigma g^{-1} \quad \text { for all } \sigma \in \Delta .
$$

TheOREM 3. Let $\Delta$ be a unitary congruence group and $\Lambda$ an automorphism of $\Delta$. Then there is a semilinear isomorphism $g$ of $V$ onto $V$ (in fact a semisimilitude) and a homomorphism $\chi$ of $\Delta$ into the elements of norm 1 in the field $F$ such that 


$$
\Lambda \sigma=\chi(\sigma) \cdot g \sigma g^{-1} \quad \text { for all } \sigma \in \Delta .
$$

Isomorphisms between congruence groups of different types. Now let $\Delta_{1}$ and $\Delta_{2}$ be any two of the above congruence groups of different type; (i.e., $\Delta_{1}$ a linear congruencegroup and $\Delta_{2}$ a symplectic congruence group, or $\Delta_{1}$ a unitary congruence group and $\Delta_{2} \cdots$ etc.). Let the associated integral domains involved all have characteristic not 2, let all dimensions involved be at least 6 , and when $\Delta_{1}$ is a unitary congr uence group suppose the hermitian form has index at least 3 .

Let $\Lambda$ be an isomorphism of $\bar{\Delta}_{1}$ onto $\bar{\Delta}_{2}$. Again we show the transvections in $\bar{\Delta}_{1}$ possess group theoretical properties no other transformations in $\bar{\Delta}_{2}$ enjoy except the transvections. It follows the isomorphism $\Lambda$ maps projective transvections to projective transvections. So we have a bijection between the lines in the underlying vector spaces of $\bar{\Delta}_{1}$ and $\bar{\Delta}_{2}$. This time since these two vector spaces possess radically different geometries, the existence of such a bijection leads to a geometrical contradiction. Thus $\bar{\Delta}_{1}$ and $\bar{\Delta}_{2}$ cannot be isomorphic; this in turn clearly implies $\Delta_{1}$ and $\Delta_{2}$ cannot be isomorphic. Thus (under the assumptions above) we prove

THEOREM 4. Let $\Delta_{1}$ or $\Delta_{2}$ be any of the linear, symplectic, or unitary congruence groups. Then $\Delta_{1}$ and $\Delta_{2}$ are not isomorphic unless they are of the same type.

Further questions. We did not determine all the automorphisms of the four-dimensional symplectic congruence groups. Over perfect fields of characteristic 2 there are exceptional automorphisms which arise from the well-known duality of the Dynkin diagram of the Lie algebra $B_{2}$.

Finally it would be interesting to settle the question of the isomorphisms between congruence groups in low dimensions. And the question of the automorphisms of the unitary congruence groups with respect to a hermitian form of index less than 3 remains unsettled.

\section{REFERENCES}

1. J. Dieudonné, La géométrie des groupes classiques, 2nd ed., Springer-Verlag, Berlin, 1963. MR 28 \#1239.

2. J. E. Humphreys, On the automorphisms of infinite Chevalley groups, Canad.J. Math. 21 (1969), 908-911. MR 40 \#1397.

3. O. T. O'Meara and H. Zassenhaus, The automorphisms of the linear congruence groups over Dedekind domains, J. Number Theory 1 (1969), 211-221. MR 39 \#4292.

4. O. T. O'Meara, Group-theoretic characterization of transvections using CDC subgroups, Math. Z. 110 (1969), 385-394. MR 40 \#1486. 
5. R. E. Solazzi, The automorphisms of certain subgroups of $P G L_{n}(V)$, Illinois J. Math. (to appear).

6. - The automorphisms of the symplectic congruence groups, J. Algebra (to appear).

7. - The automorphisms of the unitary groups and their congruence subgroups, Illinois J. Math. (to appear).

8. - On the isomorphisms between certain congruence groups (to appear).

9. - On the isomorphisms between certain congruence groups. II (to appear).

10. R. Steinberg, Automorphisms of finite linear groups, Canad. J. Math. 12 (1950), 606-615. MR 22 \#12165.

University of Notre Dame, Notre Dame, Indiana $46556^{2}$

1 The author's present address is Indiana University, Bloomington, Indiana 47401. 